Historic, Archive Document

Do not assume content reflects current scientific knowledge, policies, or practices. 


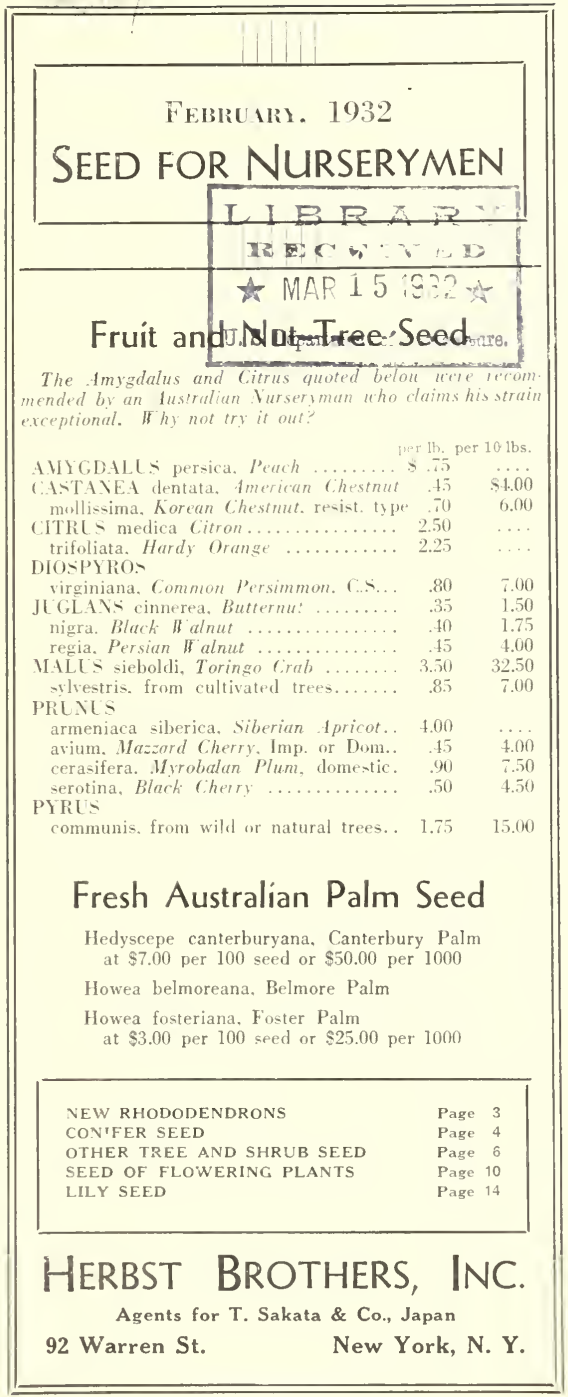




\section{HIMALAYAN RHODODENDRONS}

\section{Available only in packets of about ${ }_{16}^{16}$ ounce $\$ 1.50$ cash.}

ANTHOPOGON: A low shrub found at an altitude of 14,000 feet. Lvs. $1 \frac{1}{2}$ in. long. Fls. funnel form $1 / 2$ in. long, yellow with white terminals.

ARBOREUM: Shrub or tree attaining 40 feet, at 8/9000 feet. Found hardy in Pennsylvania. Lvs. oblong, 4-6 in. long and distinctly veined. Fls. $11 / 2$ in. long, in dense clusters, color range deep red to pinkish white.

ARBOREUM: Same as above but collected only from deep red flcwering plants.

BARBATUM: Tree to 60 feet at $12,000 \mathrm{ft}$. Lvs. elliptic 5-7 in. long. Flowers bright, scarlet $1 \frac{1}{2}$ in. across in dense trusses. Prospers in ordinary soil.

CAMELLIAEFLORUM: Six foot shrub found at 8/9000 feet altitude. Lvs. Lanceolate, 2-3 long. Flowers camellia like, 1-2 in. white faintly tinged rose.

CAMPANULATUM: Large 16 feet shrub found at 13,000 feet. Lvs. elliptic-cblong 3-6 in. long. Flowers campanulate 2 in. across vary in color from purple and blue to pale lilac white. Extremely hardy.

CILIATUM: Small 2-4 feet shrub, prospering at 10,000 feet. A wonderful rock garden subject. Lvs. elliptic about 2 in. long. Fls. in May, campanulate $1 \frac{1}{2}$ in. across, pale purple and rosy,

CINNABARINUM: Slender branched shrub at 11,000 feet. Lvs. almost Lanceolate $2-3$ in. long. Fls. A pril/May tubular, nodding, $11 / 4$ in. long, brick-red to orange.

DALHOUSIAE: Straggling eight ft. shrub found a: 8000 feet. Epiphytic in character. Lvs. elliptic 4-5 in. long. Flowers 3-7 in umbellate head. Blooms resemble the Bourbon Lilly, very large $41 / 2$ in. across and have a sweet lemon scent. Color varies from yellow and pink to creamy white, of ten spotted orange.

EDGEWORTHI: A small straggling epiphytic pendulous shrub found upon rocks and trees at $7 / 8000 \mathrm{ft}$ altitude. Lvs. obovate, blackish green, 3-4 in. long. Fls. May/June, few but large campanulate shape $4 \frac{1}{2}$ in. across 3 in. long. White tinged blush and pale yellow.

FALCONERI: Shrub or tree attaining $30 \mathrm{ft}$. According to J. D. Hooker never found under $10,000 \mathrm{ft}$. Lvs. obovate 8-10 in. long. Fls. in heads with immense numbers. campanulate 1-2 in. across, cream colored or pale yellow with dark purple blotches.

GRANDE: Tree to $40 \mathrm{ft}$. growing at $9000 \mathrm{ft}$. Lvs. oblong-obovate $8-10$ in. long $3-5$ in. wide considered the largest leaves of the specie. Fls. in April, campanulate, 5 lobed, 2-3 in. across, 2-21/2 in. long pinkish changing to white.

HODGSONl: Shrub or small tree, $20 \mathrm{ft}$. 10/12000 feet altitude. Lvs. 12-18 in. long, thick, much wider at the end than at base, brilliant deep green. Flowers May/June, copious, campanulate, 2 in. across, dark rose to pale purple.

LANATUM: Very small shrub on tree, $10 / 12000 \mathrm{ft}$. attitude. Lvs. obovate with tawny wooly tomentum beneath $3-5$ in. long. Fls. June, 610 broadly campanulate $2-21 / 2$ in. across, yellow spotted red within. A fine rock garden plant.

LEPIDOTUM: Slender $4 \mathrm{ft}$. shrub found at $12000 \mathrm{ft}$.. Lvs. obovate, glaucous $1 / 2-11 / 2$ in. long. Fls. $1-3$, campanulate, dull purple sometimes yellow, $1 / 2$ in. across.

LEPIDOTUM var. CHLORANTHUM: $2-4 \mathrm{ft}$. shrub at $7000 \mathrm{ft}$. Fls. May/June, greenish or pale yellow, spotted greenish about 1 in. across.

MADDENI: $8 \mathrm{ft}$. shrub found $6000 \mathrm{ft}$. elevation. Lvs. lanceolate 4-6 in. long. Flowers June/August, funnel shaped, $3 / 4$ in. long, 3 in. across. White, faintly blushed, agreeably scented.

NIVEUM: Small shrub allied to R. Campanulatum. Lvs. 3-6 in. long. Fls. campanulate, 2 in. across, light rosy lilac with purple spots.

SETOSUM: Shrub about $1 \mathrm{ft}$. high. Found between $11 / 16000 \mathrm{ft}$. A remarkable rock garden subject. Lvs. oblong $1 / 3$ to $1 / 2$ in. long. Fls. copious, tubular, about $1 \frac{1}{2}$ across, live rose color.

THOMPSONI: Shrub. about 15 feet. Seed collected 11/13,000 ft. Lvs, oval, 2-3 in. long, thin. Fls. 8-10, campanulate, 2 in. across, glossy bright red, calyx light green.

TRIFLORUM: $6 \mathrm{ft}$. shrub, found at 7/13,000 feet. Ovate $2-3 \mathrm{in}$. long. Fls. shaped like common azalea, May/June, greenis yellow, 3 in. across.

WIGHTI: Shrub to 14 feet. 12,000 feet altitude, Lvs. 6-8 in. long. Fls. rich, yellow, spotted red within, campanulate, $2 \frac{1}{2}$ in. across.

WIGHTI: Same as above, however, only one plant found at 13,000 feet with white bell shaped flowers, spotted crimson. Only very few packets available. 


\section{Terms}

This list cancels all previous offers.

All sales are made subject to the terms and conditions of our printed catalog.

Disclaimer: We give for ourselves or our Principals no warranty, express or implied, as to the description, quality or productiveness, or in any other respect for the seed we deliver, nor will we be responsible for the crop in any way. In no case shall we or our Principals be held liable for more than the invoice value of the seed sold.

Payment: Our prices are calculated on a strictly cash basis; customers remitting with their orders will be allowed $5 \%$ discount. When remitting please add for postage and packing.

Less than 8 ozs. furnished at an advance in price of $25 \%$; minimum order. unless specifically provided. 4 ozs. Minimum charge for any one item 25 cents.

seed of Oriental origin is offered for account of Messs. T. Sakata \& Co.. Japan's Leading Seedsmen. As their represen. tatives in the United States and Canada you will always find us prepared to quote and assist you in locating seed originating in the Far East.

\section{Japanese Flowering Cherries}

Have arrived from Japan

The late Dr. E. H. Wilson continually urged the use of Japanese understock for the Oriental Flowering Cherries, in spite of the difficulty of their production from seed. Too often failure in germination is attributed to the quality of the seed rather than to faulty handling. Check tests through botanical institutions convince that our method of packing the seed for the long trip from Japan brings it to our customers in prime condition. Our new crop seed is now in New York. If we have your order it is immediately dispatched to you the day your order is received. The seed should AT ONCE be put in flats with moist sand. The sand must never be allowed to dry. The flats may be kept in the open where they are subject to natural stratification. In warmer climates artificial stratification is necessary as cherries require at least 12 weeks to afterripen. Sowing in the open is advisable as soon as the weather permits. One successful grower sowed his seed in a two inch deep furrow, filling it over with grain husks. This reduced the weed probtem and helped supply the seed its continual demand for moisture.

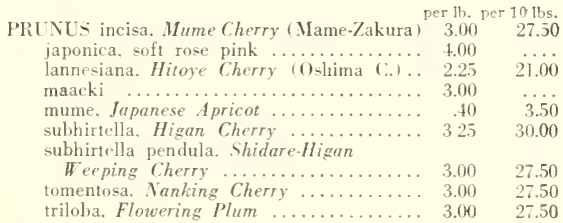




\section{ABIES Conifer Seed}

$\begin{array}{cc}3.00 & 27.00 \\ . & 3.60\end{array}$

cicilica, Cicilian Fir ................... $3.60 \quad 32.00$

concolor, White Fir .................. $1.50 \quad 12.00$

firma, Momi Fir, grows rapidly......... $1.25 \quad 11.50$

homolepis (brachyphylla) ........... $2.00 \quad 17.50$

lasiocarpa. Alpine Fir ............... $3.00 \quad 27.00$

mariesi, Maries Fir ................ 2.50 22.50

nordmanniana, Nordmann Fir ........ $2.50 \quad 22.50$

pectinata, Silver Fir ................ $1.25 \quad 11.00$

sachalinensis, Sakhalin Fir, perfectly hardy $\quad 2.75 \quad 25.00$

veitchi, Veitch Fir ................. $2.60 \quad 23.00$

CEDRUS atlantica, Atlas Cedar .......... $3.00 \quad 27.00$

atlantica argentea ................. $3.25 \quad \ldots$.

atlantica glauca, Blue Atlas Cedar....... $4.50 \quad 40.00$

deodora, Deodar Cedar ............... $3.50 \quad 32.50$

libani, Cedar of Lebanon ............. $3.00 \quad 27.00$

CEPHALOTAXUS

drupacea, Japanese Plum Yew........... $\quad .75 \quad 6.50$

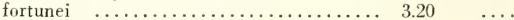

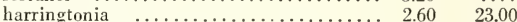

CHAMAECYPARIS lawsoniana, Lawson C... $2.50 \quad 22.00$

lawsoniana argentea ............... 4.50 40.00

nootkatensis, Nootka Cypress ......... 6.00

obtusa, Hinoki Cypress ............... $1.25 \quad 11.50$

obtusa var. breviramea .............. $2.50 \quad \ldots$.

pisidera, Sawara Retinospora ............ $1.25 \quad 11.50$

pisifera plumosa .................. $8.00 \quad \ldots$

CRYPTOMERIA

japonica elegans. Plume $C$. ......... 5.50

japonica lobbi, Lobb $C$. ............. 4.00

CUNNINGHAMIA

lanceolata, China Fir .............. $3.00 \quad 27.50$

CUPRESSUS

arizonica, Arizona Cypress ............ 2.25 20.50

macnabiana, Macnab Cypress ........... $4.50 \quad 40.00$

GINGKO

Italian Cypress ................. 2.00

biloba. Maidenhair Tree ................ .45 4.00

JUNIPERUS chinensis, Chinese Juniper, C.S.. $2.25 \quad 20.00$

communis, Common Juniper, C.S......... $1.75 \quad 16.00$

communis depressa, Prostrate Juniper, B. $1.50 \quad 13.50$

excelsa. Greek Juniper, C.S. ........... $2.75 \quad 25.00$

horizontalis douglasi, Waukegan Juniper,

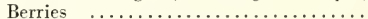

japonica, Japanese Juniper, C.S......... procumbens, Hardy Chinese Creeping, B.. sabina, Savin Juniper, C.S.............. scopulorum, Colorado Juniper, Berries.... virginiana, Red Cedar, (Platte River) B... virginiana, Red Cedar (Northern), Berries

$2.50 \quad 22.50$

$3.00 \quad 27.50$

$3.50 \quad 32.50$

$4.00 \quad 36.00$

$1.50 \quad 13.5 n$

$1.00 \quad 7.50$

$1.35 \quad 12.00$

$1.50 \quad 13.50$

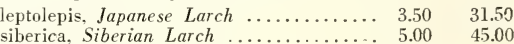

LIBOCEDRUS

decurrens, California Incense Cedar .... $2.00 \quad 18.00$

PICEA canadensis, IT hite Spruce .......... 2.50 22.50

engelmanni. Engelman Spruce ........ $4.00 \quad 36.00$

excelsa. Norway Spruce .................. $1.25 \quad 11.50$

glauca albertiana. Black Hills Spruce... $4.50 \quad 41.00$

jezoensis hondoensis. Hondo Spruce..... $350 \quad 32.50$

mariana, Black Spruce .............. $8.50 \quad 78.00$

orientalis. Oriental Spruce ............ $10.00 \quad \ldots$.

pungens. Colorado Spruce ............. $4.50 \quad 42.00$

sitchensis, Sitka Spruce ............. $4.75 \quad 42.50$ 


\section{CONIFER SEED Cont'd}

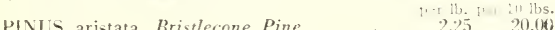
aristata, Bristlecone Pine ........ 2.25 20.00 bungeana, Lacebark Pine .............. $2.00 \quad 18.0 \mathrm{C}$ canariensi-, Canaty Island I'ine igenuinel $2.00 \quad 18.0$ ') cembra, Swiss Stone Pine ............ $90 \quad 8.00$ densiflora. Japanese Red Pine ....................... $1.25 \quad 6.00$ echinata. Short Leaf Pine ............ $6.00 \quad 54.0$ ? edulis, Nut Pine .................. $1.00 \quad 9.00$ excelsa, Himalayan Pine ............ $3.00 \quad 27.00$ flexilis, Limber Pine ............... $2.50 \quad 22.50$ halepensi. . Aleppo Pine ................ $1.70 \quad \ldots$

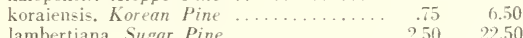

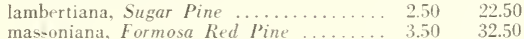
monophylla, Singleleaf Pine ........... $4.75 \quad 43.50$ montana. Swiss Hountain Pine ......... $2.50 \quad 22.50$ montana mughus, Hugho Pine ........... $2.50 \quad 22.50$ montana uncinata, Large Swiss Pine.... $3.00 \quad 27.00$ monticola. Western Wlite Pine ......... $5.00 \quad 45.00$ murrayana, Lodgepole Pine ............ $6.75 \quad 62.50$ nigra, Austrian Pine ................. $1.80 \quad 16.00$ nigra poiretiana. Corsican Pine igenuine) $3.25 \quad 28.00$ palustris, Longleaf Pine .............. $2.50 \quad 22.50$ parviflora (pentaphylla). Japanese If hite

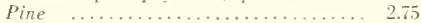
peuce, Macedonian Pine ............. 3.50 pinea, Italian Stone Pine ............. 9.90 ponderosa. II estern Yellow Pine (Colo.).. 1.75 pumila. Creeping Pine ............... 2.75 rigida. Pitch Pine ................. 6.50 strobus, IT hite Pine .............. 250 sylvestris. Scots Pine ............... 250 sylvestris rigensis, Riga Scots Pine..... 3.50 sylvestris, H. B. Special ............ 5.00 tabulaeformis. Chinese Pine .......... 1.50 taeda, Loblolly Pine ................ 525 tanyosho, Japanese Table Pine.

true seed .......... per $1 / 41 \mathrm{lb} . \$ 5.50 \quad 17.50$ thunbergi, Japanese Black Pine......... 1.25

PODORARPT: chinensis ............ 1.50

PSELDOTSLGA douglasi glauca. Colorado Douglas Fir ................. $5.50 \quad 50.90$

SCIADOPTYS verticillata. T'mbrella Pine... $2.75 \quad 2500$

\section{SEQUOIA}

sempervirens. California Redwood ...... 2.50

TAXODIU II distichum. Common Baldcypress. 1.00

TAXI - baccata, English Yew .......... $227 \quad 201$. baccata adpressa, Shortleaf English Iew.. $3.60 \quad 33.50$ cuspidata. Japanese Yew ............ $1.25 \quad 1109$

THLJA occidentalis. American Arbortitaf.... $3.50 \quad 37.50$ occidentalis wareana. II are Arbortitac.... $5.50 \quad 500$ orientali.. Orientai Arborvitae............. 1.00 900 orientalis aurea. Golden Orientul 4..... 2.75 orientalis compacta. Nuarf Oriental A... $225 \quad 20.00$ orientalis sieboldi. Siebold Arborvitae.... 2.75 25.00

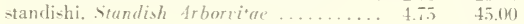

THL JOPSI dolabrata. False frborritae.... $250 \quad 22.50$

TORREY A nucifera. Japanese Torreva.

large edible nuts .............., $1.00 \quad 9.00$

TSLG.A canadensis. Canadian Hemlock ...... $7.00 \quad 63.00$ canarlensis. NEW ENGLAXD .EED ..... $9.50 \quad \ldots$. 


\section{Other Tree and Shrub Seed}

ACER dasycarpum, Silver Maple (1932 Crop . palmatum, Japanese Maple ............ $1.65 \quad 15.50$ palmatum atropurpureum, Bloodleaf

Japanese Maple ....... per 1/4 ib. $\$ 3.00$ paimatum atropurpureum dissectum,

Threadleaf Red Maple.. per 1/4 lb. $\$ 5.00$ platanoides, Norway Maple .............. pseudoplantanus, Sycamore Maple ....... rubrum, Red Maple (1932 Crop)......... saccharum, Sugar Maple .............

AESCULUS

hippocastanum, Horsechestnut ........ .45

turbinata, Japanese Horsechesinut ........

AKEBIA quinata, Fiveleaf Akebia ......... 2.00

ALNUS glutinosa. European Alder .............. japonica

AMELANCHIER canadensis, Downy Shadblow, Berries..$\ldots \ldots \ldots \ldots \ldots \ldots \ldots \ldots$.

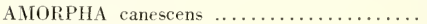

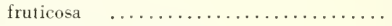

AMPELOPSIS quinquefolia $\ldots \ldots \ldots \ldots \ldots$

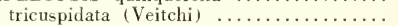

ARALIA chinensis, Chinese Aralia, C........ spinosa, Devil's-W alkingstick, C.S........

ARCTOSTAPHYLOS glauca, Great

Manzanita $B . \ldots \ldots \ldots \ldots \ldots \ldots \ldots . \ldots \ldots . \ldots \ldots$ uva ursi, Bearberry ................... 1.10

ARONIA arbutifolia, Red Chokeberry B.... $\quad .90$

ASIMINA tribola, Papaw ............... 1.50 $\ldots$

$\cdots$

AZALEA kaempferi ... \$2.00 oz. $\$ 7.001 / 4 \mathrm{lb}$. mollis, C.S. ........ $75 \mathrm{oz} . \quad 2.00 \mathrm{1} / 4 \mathrm{lb}$. mucronulatum, rose colored, C.S. .... \$1.50 oz. nudifolia, Pinxterbloom C.S. $\quad . . . \cdots \cdots . . .1 .00 \mathrm{oz} . \quad 3.50 \mathrm{I} / 4 \mathrm{lb}$. occidentalis, Western Azalea, C.S. ....... $1.50 \mathrm{oz} .4 .50 \mathrm{I} / 4 \mathrm{lb}$. poukhanensis, Korean Azalea, C.S. ..... $3.50 \mathrm{oz}$. quinquifolia rosa, soft pink flowering, C.S. $3.501 / 2 \mathrm{oz}$. schippenbachi, Royal Azalea, C.S. ...... $2.50 \mathrm{oz} .7 .50 \mathrm{I} / \mathrm{lb}$. vaseyi, Pinkshell Azalea,

C.S. ........... $2.00 \mathrm{oz}$.

BENZOIN aestivale, Spicebush ........... 1.10

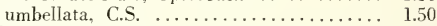

BETULA alba. European White Birch........ alba pendula, European Weeping Birch... populifolia. Gray Birch .................

BIGNONIA radicans, Trumpetcreeper .........

BUDDLEIA davidi, Orange-eye .............

BUXUS koraiensis, Hardy Korean Boxwood... sempervirens $\quad . \ldots \ldots \ldots \ldots \ldots \ldots \ldots$.

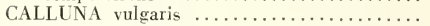
occidentalis, California Sweetshrub ..... 3.75 


\section{Herbst Brothers, Ing.}

CABLE ADDRESS HERBST - NEW YORK

A. B. C. S, IMP. \& 6 , RUD. MOSSE

BENTLEY, RIBEIRO

To the

Practical Forester

and Nurseryman

\section{WARREN STREET \\ NEW YORK}

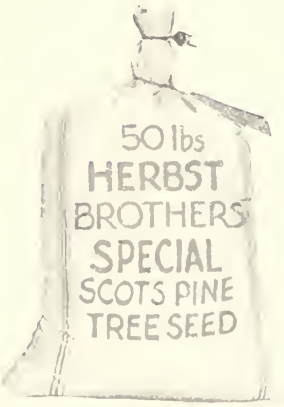

Shipped only in sealed bags Price $\$ 5.00$ per pound
The success of all plantings of conifers depends initially on the source of seed used. Your disappointments in tne past need never be repeated in the case of Pinus sylvestris if you will specify our "HERBST BROTHERS' SPECIAL SCOT'S PINE SEED".

This folder tells why in a simple, straightforward way. We hope that you will examine the reproductions of the photos. Their story will convince you.

\section{Sincerely yours,}

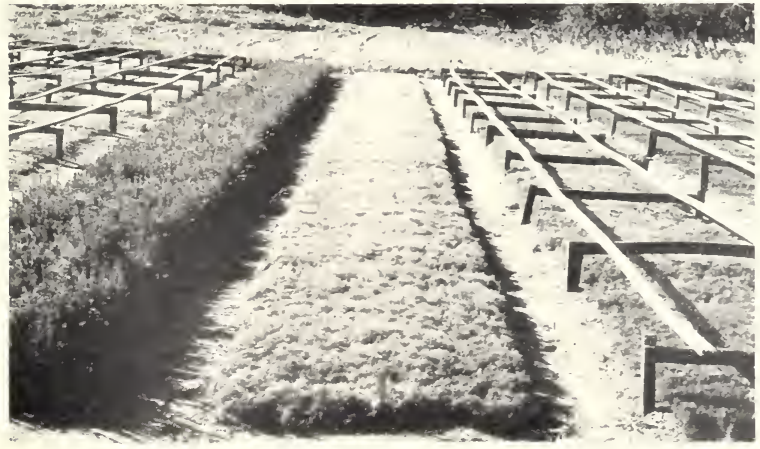

Photo by Courtesy of Ohio Agricultural Experiment Station, Wooster, Ohio
ONE-YEAR SCOT'S PINE SEEDLINGS grown from "H. B. SPECIAL SEED"

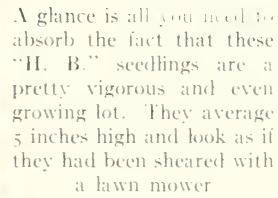




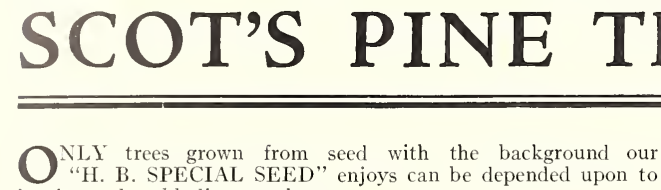
inspire such noble lines as these:

$$
\begin{aligned}
& \text { "Regal and stately behold it stand } \\
& \text { Above its brethren, towering grand, } \\
& \text { A sentinel guarding the sleeping land. } \\
& \text { Beauty and grace in its form combine } \\
& \text { A monarch, born of a noble line, } \\
& \text { Long may it be, ere its race decline! } \\
& \text { Frost shall not wither a leaf of thine, } \\
& \text { Fearless and fadeless Pine!" }
\end{aligned}
$$

The nurseryman seeks a vigorous, well-shaped tree. The problem of the practical forester is to produce for the future a stand of marketable trees which will give the greatest possible return on the investment which extends over several decades before profits can be collected. Neither can afford to take chances on the seed-only the very best justifies their undertaking.

The pictures on this page show what happens when scientifically grown seed is planted-also where seed of unknown lineage is used.

\section{SEEDS}

from

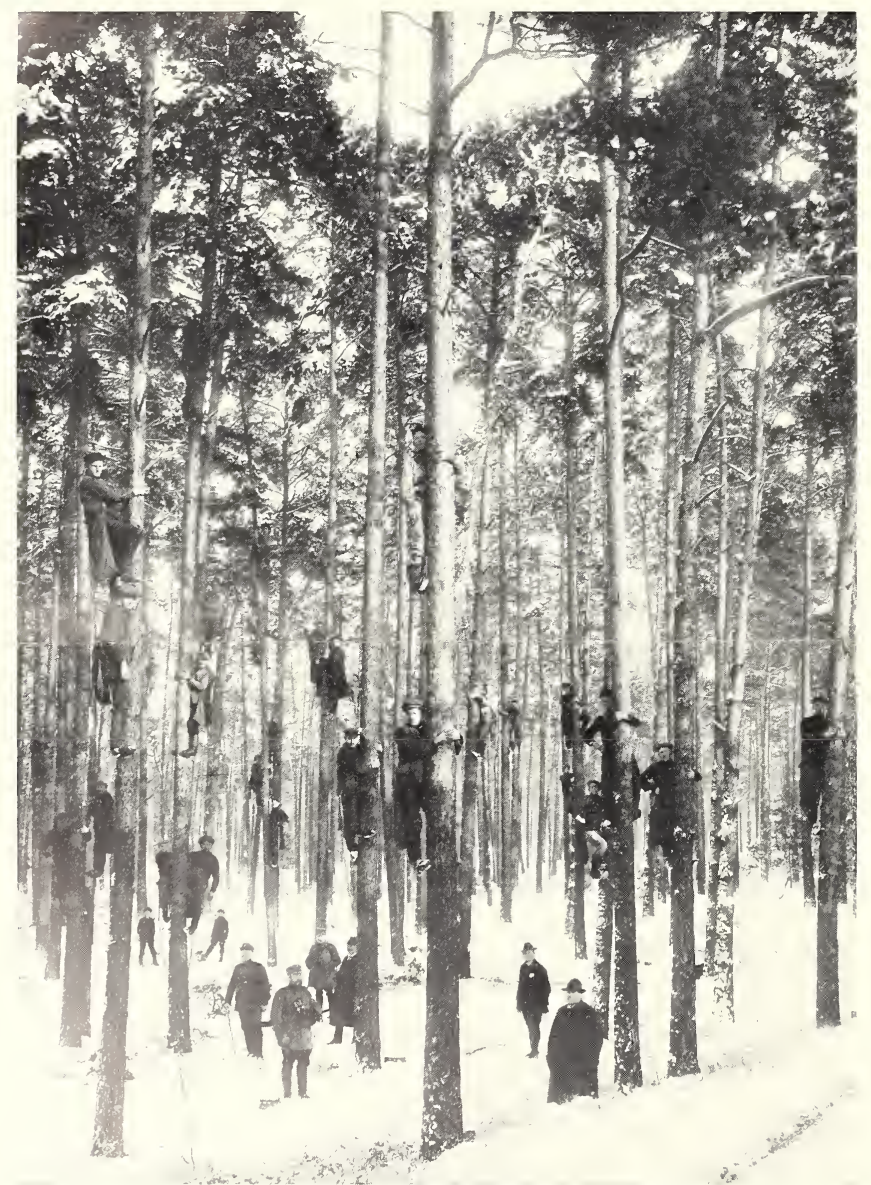

\section{FOREST \\ SCENE where \\ SEEDS are \\ GATHERED \\ from \\ STRAIGHT- \\ GROWING \\ TREES}

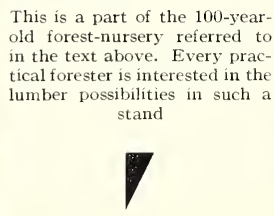

A

CLOSE-UP

of $a$

TYPICAL SEED-

\section{GATHERER}

Please remember that ALL "H. B. SPECIAL SEED" are gathered from trees-never from the ground. The climber's equipment consists of: half-bushel cone-bag slung across the shoulder; a lightweight 8 to 10 foot pole with a hooked end for drawing in the cone-bearing branches for ease in picking; and the usual lineman's climbers

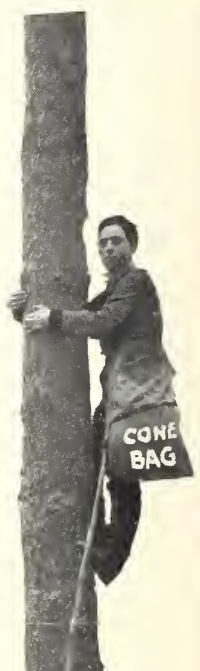

Griman Pine Forest Plantation-Source of OUR Seeds a section of a German forest where "H. B. Note please the perfect cons of gathering seeds. Trees are climbed and the stem-ripened cones collected by hand
from the tree itself-NONE FROM THE GROUND. This stand is tut a small part ot a large acreage isolated under governmental ences and devoted to this these priceless heritages-straight-growing habit and immense

In 1772 a group of farsighted German foresters recognizing the tremendous reforestation and ornamental values of Scot's Pine (Pinus sylvestris) undertook to produce a variety which could be 


\section{RAIGHT-GROWING ANCESTORS!}

relied on to grow straight as well as rapidly. All Europe was searche for parents that measured up to this standard and seed secured.

This problem was an investment in decades - yes, in centuriesperiod too long for one set of individuals to control. Igain, the menace was ever present that cross-pollenization from inferio varieties of Scot's Pine might ruin the work. So the control of the area was vested in the municipalities governing the locations ideal for the purpose. Local laws prevented others from planting scot's Pine within the districts set aside. Thus effectual protection has been perpetual. The results have justified the methods.

$$
\begin{aligned}
\text { RIGID ELIMINATION OF UNDESIRABLE } \\
\text { SPECIMENS OF PINE }
\end{aligned}
$$

Regular plantings were established at clefinite periods in rotation to insure in subsequent years a continuous crop of seed from trees in their prime. While these seed plots were growing they received extra critical attention from experienced foresters who eliminated. first from seedlings, then from older plants, all individuals that failed to reach the predetermined standards of growth rapidity. straight habit. resistane to weather and disease, and density and inspected their bark.

secided on were permi
exceptional conditions

HERBST BROTHER'S SPECIAL SCOT'S PINE TREE SEED

is gathered the product of the most pain-taking tren

the last 200 years of practical forestr.

A TRIBUTE FROM FRANCE

\section{Crooked Scot's Pine}

\section{Years Old}

\section{Produced from Seed}

Gathered from

"ANYWHERE and

\section{EVERYWHERE"}

The hopelessness of trees such as these

is obxious even to a layman. They will

never pay for the investment they re

present. You can prevent recurrences

of misfortunes of this kind -

\section{Plant H. B. Special Scot's Pine Seed}

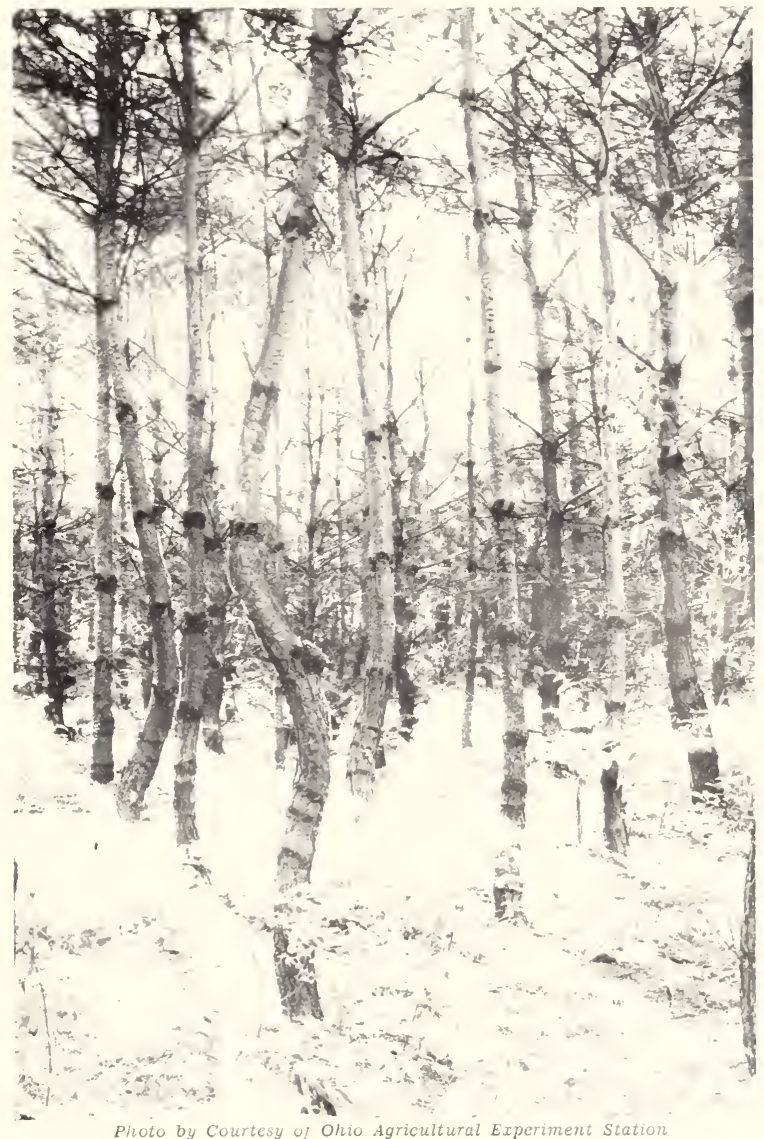




\section{HERE'S PROOF of the SUPERIOR GROWING QUALITIES OF HERBST BROTHERS, "SPECIAL" SCOT'S PINE TREE SEED}

FVERY season more leading Foresters and Nurserymen are becoming convinced that as any grown in Europe-just as quickly too. Its increasing prestige becomes more widespread every year. In all experiments it has produced perfectly straight stems and almost twice the height of other types in the same time. Please note the comparisons below.
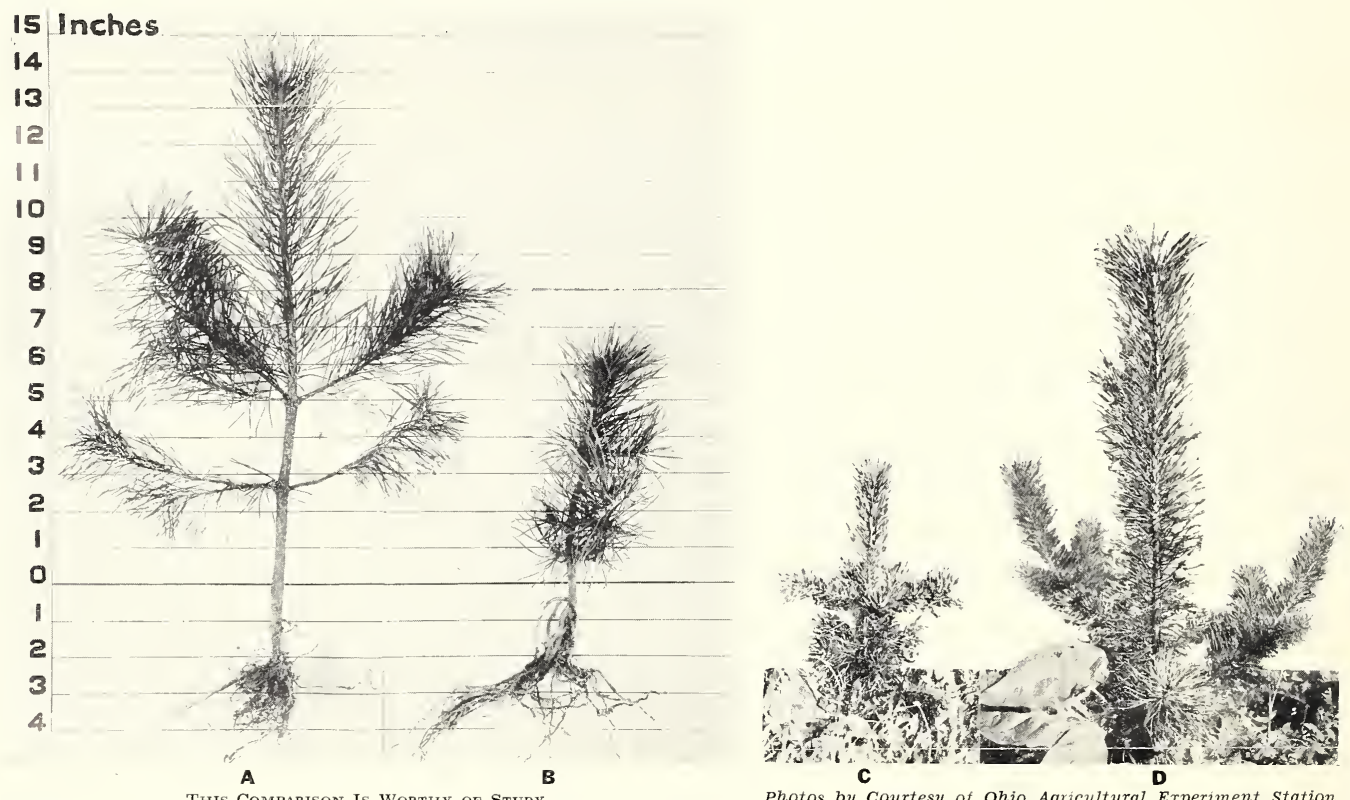

This Comparison Is Worthy OF Study

Photos by Courtesy of Ohio Agricultural Experiment Station

\section{EXPLANATION OF ABOVE DIAGRAM AND COMPARISONS}

Compare $A$ with $B$-both 4 -year Transplants

"A" is from "H. B. SPECIAL SEED"

Compare $C$ with $D$-both 5-year Transplants

"D" is from "H. B. SPECIAL SEED"

"C" and "D" are both forest plantings on permanent "location."

Germination: Every ounce of "H. B. SPECIAL SEED" is taken from hand-picked cones which have been individually appraised by experienced experts. It is extracted most painstakingly so that we may honestly maintain that it is of the highest germination and viability procurable.
General Data: These trees grow from 65 to 98 feet high and attain an age of 66 to 120 years.

Knots usually on upper third of trunk-lower two-thirds clear. Needles 2 3-10 inches to 3 I-IO inches long-borne in very heavy clusters.

Color of needles; deep green from September to April-a tritle lighter from May to August.

Seedlings reach a height of 4 to 6 inches in first year-growth thereafter, I I to 25 inches yearly. -

Provenience: "H. B.SPECIAL" is collected at about 300 feet above sea level.

Longitude: I 2 East-Latitude: 52 North.

Temperature: Maximum, 104 deg. F.-Minimum, 22 deg. F.

Precipitation: 20 to $23 \frac{1}{2}$ inches.

Frost Free Months: Six. 
OTHER TREE AND SHRUB SEED - Cont'd

Catalpa per lb. per $10 \mathrm{lbs}$

... $1.10 \quad 10.00$

CEANOTHUS americanus, Jersey-tea, in hull. $1.10 \quad 10.60$

CELASTRU - scandens, American Bittersweet,

Berries ..................... $1.50 \quad 13.50$

CELTIS australis. European Hackberry ..... $.95 \quad$....

CERCIS chinensis, Chinese Redbud ........ $3.75 \quad 35.00$ occidentalis, Calitornia Redbud .......... $3.00 \quad 27.00$

CHIONANTHL - virginica. It hite Fringe.... $3.75 \quad 35.00$

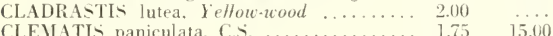

virginiana, C.S. . . . . . . . . . . . . . $4.00 \quad 36.00$

vitalba $\ldots \ldots \ldots \ldots \ldots \ldots \ldots \ldots \ldots \ldots \ldots$

viticella. Atatian Clematis ............ $1.50 \quad 13.50$

CLETIIRA alnifolia. Summersweet ........ $150 \quad 13.50$

CORNL's alternifolia. Pagoda Hogwood. B.... $1.50 \quad 13.50$

amomum, Silky Dogwood. B. .......... $1.50 \quad 13.50$

canadensis, Bunchberry .............. $2.00 \quad 18.00$

florida. Flowering Dogwood ............ $.75 \quad 5.00$

kousa. Kousa Dogwood, C \. . . . . . . . $2.45 \quad 22.50$

nuttali. Pacific Dogwoood ............. $1.75 \quad 15.00$

sanguinea, Bloodtwig Dogwood, C.......... $1.15 \quad 10.50$

stolonifera. Red Osier Dogwood .......... $1.50 \quad 13.5^{\prime}$ )

CORYLLS americana. Imerican Hazetnut. C.. $.70 \quad 6.50$

COTONEASTER acutifolia. Pelin Cotoneaster $2.00 \quad 18.00$

horizontalis, Rock Cotoneaster.......... $3.25 \quad 29.00$

microphylla, Rockspray ............. 3.50 3250

CRATAEGUS coccinea, Thicket Hawthorn ... $1.25 \quad 11.50$

crusgalli. Cockspur Thorn ............ $1.00 \quad 9.00$

monogyma, Engfish Hawthorn. C......... $1.50 \quad 13.50$

oxycantha, English Hawthorn. C.S........ $1.00 \quad 9.00$

prunifolia. Plumteaf Hawthorn. f....... $1.00 \quad 9.00$

prunifolia. Plumteaf Hauthorn. Berrit.. .50 ...

punctata. Dotted Howthorn ............ $1.25 \quad 11.50$

CYDONIA japonica. Japanese Quince ...... $3.50 \quad 32.00$ oblonga. Common Quince ........... $2.50 \quad 22.50$

CYTISUs nigricans, Spike Broom ......... $7.00 \quad \ldots$.

DAPHNE mezereum. February Daphne. C..... $7.00 \quad 63.00$

ELAEAGNUS angustifolia. Russian Olive... $1.00 \quad 5.00$ longipes, Cherry Elaeagnus ......... $3.00 \quad 27.00$

EUONYMUS alatus. Winged Euonymus, C.... $1.25 \quad 11.57$ americanus, Brook Euonymu.s ......... $1.25 \quad 11.50$ oxyphylla, C...................... $2.25 \quad 21.50$

EXOCHORDA grandiflora, Common Pearlbush $\quad 3.50 \quad 31.50$

F.GUS americana. American Beech ........ $1.25 \quad 11.50$ sylvatica, European Beech ........... $\quad .95 \quad 7.75$ sylvatica purpurea, Purple Beech ....... $2.50 \quad 22.50$

FRAXINU's americana, White Ash ....... $90 \quad 90.25$ lanceolata, Green Ash .............. $95 \quad 8.60$

GLEDITSIA japonica, Japanese Honeylocust. . $\quad 1.50 \quad 13.50$ triacanthos. Common Hone'soc'ts. ... ... . $90 \quad 8.25$ triacanthos inermis. Thornless Honelocust 1.60$) \quad 14.75$

H.LESIA tetraptera, Great Silverbell ........ $1.25 \quad 11.00$

H IIA IEII- virginiana, Common II itch-Hazel $1.75 \quad 16.50$

HEDERA helix. Engtish Ivy. C........... $1.50 \quad 13.50$

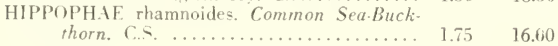

HOVEYIA dulcis. Raisentree ............ 1.00

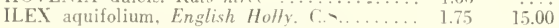

crenata. Japanese Holly. C........................ 175

glabra. Inkberry . . . . . . . . . . . . . 90.25

opaca $\quad \ldots \ldots \ldots \ldots \ldots \ldots \ldots \ldots \ldots \ldots \ldots . .9 \ldots \ldots$

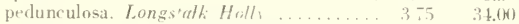

verticillata. Common $\mathbb{H}$ interbers ....... $1.25 \quad 11.50$

KALUIA latifolia. Mountain Laurel.

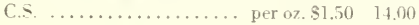


OTHER TREE AND SHRUB SEED - Cont'd per $1 \mathrm{~b}$. per $10 \mathrm{lbs}$.

KERRIA japonica, C.S. ................ 2.00

KOELREUTERIA paniculata, Golden-rain Tree $1.25 \quad$ 11.00

LABURNUM alpinum, Scotch Laburnum.... $2.00 \quad 18.00$

vulgare, Goldenchain ................ $1.50 \quad 13.50$

LAGERSTROEMIA

indica, Conmon Crape Myrtle, pink fl.... $4.00 \quad \ldots$.

white flowering ................... $5.00 \quad \ldots$

LAUROCERASUS lusitanica, Portugal Laurel $1.50 \quad 13.50$

LEUCOTHOE catesbaei, Drooping Leucothoe, C.S. ........... oz. $\$ 2.00$ I $/ 4$ Ib. $\$ 6.00$

LIGUSTRUM ibota, C.S. ................. japonicum, Japanese Privet ............ vulgare, European Privet .............

LIQUIDAMBER styraciflua, Sweetgum ..... 3.20

LIRIODENDRON tulipifera, Tulip Tree ..... 1.25

LONICERA maacki, Amur Honeysuckle...... 4.00

MIAGNOLIA acuminata, Cucumbertree ...... 2.00

grandiflora, Southern Magnolia ......... 2.00

stellata, Star Magnclia .............. 3.50

lennei, domestic, C.S. .............. 4.00

tripetala, Umbrella Magnolia ......... 2.00

MAHONIA aquifolium, Oregon Hollygrape, C.S.

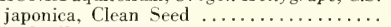

MORUS alba tatarica ..................

MYRICA carolinensis, Northern Bayberry, B.. cerifera, Southern Waxmyrtle, B.........

NANDINA domestica, Berries ..............

NYSSA sylvatica, Tupelo .................

OXYDENDRUM aboreum, Sourwood, in hull.

PALIURUS spina-christi, Christ Thorn ......

PITTOSPORUM

undulatum. Orange Pittosporum .........

PLATANUS occidentalis, American Planetree orientalis, European Planetree ...........

POPULUS tremula, European Aspen ........

PTEROCARYA rhoifolia, Japanese Wingnet..

PYRACANTHA coccinea lalandi, C.S........

$3.00 \quad 27.00$

3.2530 .00

$2.50 \quad 22.50$

$1.80 \quad 16.00$

$1.60 \quad \ldots .$.

$\begin{array}{ll}1.10 & 10.00\end{array}$

$1.30 \quad 11.50$

$1.25 \quad 11.00$

$1.15 \quad 10.50$

$2.90 \quad 26.00$

$\begin{array}{ll}.85 & 7.50\end{array}$

$\begin{array}{ll}.80 & 7.20\end{array}$

$1.50 \quad 13.50$

$1.25 \quad 11.50$

\section{QUERCUS}

alba, White Oak ......... per $\mathrm{lb}$.

cerris, European Turkey Oak.....

coccinea, Scarlet Oak .......... .25

ilex, Holly Oak .................75

lyrata, Overcup Oak ..................

nigra, Water Oak ............. . .30

palustris, Pin Oak ................. 30

phellos, Willow Oak ..............35

prinus, Chestnut Oak ............ .45

robur, English Oak ...............40

rubra. Common Red Oak ........ .25

sessiflora. Durmast Oak ........... .60

stellata, Post Oak .............. .25

velutina, Black Oak ............ .25

Ask

for

prices

on

quantities

RHAMNUS alaternus, Italian Buckthorn, C.S.. alpina, Alpine Buckthorn. C.S. .......... cathartica, Common Buckthorn. C.S. ..... frangula, Glossy Buckthorn. C.S. ........ purshiana, Cascara Buckthorn, B.........

per lb. per $10 \mathrm{lbs}$.

$2.00 \quad 18.09$

1.7516 .00

$1.75 \quad 16(; 0$

$1.30 \quad 11.50$

$3.50 \quad 33.50$

RHODODENDRON brachycarpum,

hardy white flowering, C.S.... oz. $\$ 2.50$ californica, Coast

Rhododendron, C.S. oz. $\$ 1.50$ I/4 lb. $\$ 5.00$ caroliniana. Carolina Rhododendron

Pods ................... I I/4 lb. $\$ 2.00$ catawbiense, Catawba Rhododendron

oz. $\$ 1.40 \mathrm{I} / 4$ lb. $\$ 4.75$

chrysantheme' $m$. C.S. ....... 1/4 oz. $\$ 3.00$ 
OTHER TREE AND SHRLB SEED-Cont

RHODODENDRON-Cont"d

hybrids, mxd. C.S. I 4 oz. $\$ 2.50 I_{2} I_{2} 0 z . \$ 4.50$

heiskei. C............... + (re. 83.00

maximum, Rosebay Rhododendron.

C.S. ........... oz. $\$ 1.70$ 1/4 lb. $\$ 5.50$

metlemichi. Leatherleat Rhodo-

dendron. (... pink ......... o . $=4.00$ ponticum. Pontic Rhododendron.

C.S. ......... oz. $\$ 1.30$ I $+1 \mathrm{~b} . \$ 4.00$

RHL - cotinus. Comnon sinotietree in lull- per tb. per ? ith typhina, Staghorn, suntac in hulls...... $\quad 85 \quad-\frac{85}{7.70}$

RIBE.

aureum. Slender Golden Currant, C...... 10.00

aureum, Berries ................. 4.00

sanguineun. It inter Cumant ......... 5.0.

$.75 \quad 7.00$

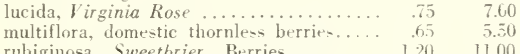

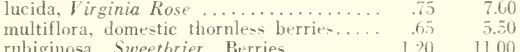

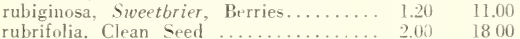

wichuriana, Wichurian Rose. C........ $1.20 \quad 11.00$

SAMBLCL \& canadensis, American Elder. B... $1.60 \quad 14.50$

SASSAFRAS variifolium, Common Sassatras.. $1.20 \quad 11.00$

-CHIZOPHRAGMA hydrangeoides, Japanese

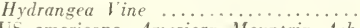

SORBUS americana. American Mountain Ash.

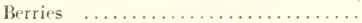
aria, White Beamtree, C... .......... aucuparia. European . Nountain Ash, Berries STERCLIIA p'atanifolia. Chinese Parasoltree

-TEWARTIA monadelpha $(. . . . . . \ldots \ldots .$.

sTYRAX obasia. Fragrant snouball, C....... 3.50

SYRINGA dilatata, C.S. ......per I/4 lb. $\$ 3.0010 .00$

FIIPLOCOC paniculata icrataegoides).

Asiaric sureetleat ................ 3.00

$7.00 \quad 65.00$

$1.15 \quad 10.50$

$1.25 \quad 11.00$

$1.00 \quad 9.00$

$1.25 \quad 11.50$

$00 \quad 36.00$

...

$\cdots$

27.50

japonica. Japanese Tree Lilac. C...

perfectly hardy ............. oz. 1.50 persica, Persian Lilac. C.S. per I/4 1b. $\$ 2.00$ vulgaris, Common Lilac, C.S. ...........

TAUARIX gallica. French Tamarix .........

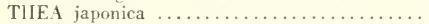

TILIA americana, American Linden. Berries.. americana macrophylla, Bigleaf American

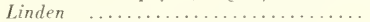
cordata. Littleleaf European Linden...... platyphyllos. Bigleaf European Linden...

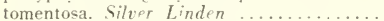

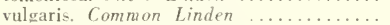

ILVIS americana. American Elm ......... pumila, Df. Asiatic Elm ............ 2.50

$3.50 \quad 31.50$

$2.00 \quad 18.00$

$.70 \quad 6.00$

$1.25 \quad 11.50$

$1.40 \quad 13.00$

$1.20 \quad 11.00$

1.10 10.00

$1.00 \quad 9.30$

$1.25 \quad 11,00$

$1.00 \quad 9.00$

22.50

VIBURNE II acerifolium. Mapleleaf

Tiburnum, B. ................... $90 \quad 800$

anericanum. Ameican Cranberrbush. B. $12 ; \quad 11.57$

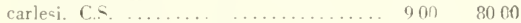

cassinnides. II itherod. B. ....................... $1.25 \quad 11.00$

dentatum. Arrowwood. B .................. $1.25 \quad 11.00$

dilatatum. Linden Viburnum. C........ I 16.5 15.0n

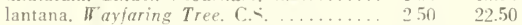

lentaqo. Vannyberry. B. ............... 1 $25 \quad 11.00$

odoratissimum. Sue I iburnm (C...... $275 \quad 25.00$

opulus, Emropean Cranberty. C......... $2.50 \quad 22.50$

prunifolium. Blacthaw. B. ............ $1.50 \quad 13.5 n$

sarrenti. Sargent Cranberrybush. C....... $2.75 \quad 2500$

sietoldi. Siebold Viburnum. C... ........ 2.75 25.00

tomento:um. Doublefile liburnum. C - ... 2.50 22.50

ITTEX ånus ca-tus Lilge Chastr Tlep...... $1.50 \quad 13.50$ 
OTHER TREE AND SHRUB SEED-Cont'd per lb. per $10 \mathrm{lbs}$.

VITIS coignetiae, Gloryvine, C.S.

(V. kaempferi) ..................... $1.35 \quad 12.00$

WISTERIA frutescens, American Wisteria.... $3.50 \quad 31.50$

sinensis, blue .................... $1.00 \quad 8.60$

sinensis, white ................. $2.00 \quad 17.50$

sinensis multijuga, blue ............. $3.25 \quad 29.00$

ZELKOVA serrata (acuminata), Sawleaf Zelkova $2.00 \quad 18.00$

\section{Seed of Flowering Plants}

The limited demand for many rare types of Flower Seed makes publication of prices unprofitable. If you do not find quoted varieties you need, please write us particulars with common and botanical names and quantities required.

\section{ACHILLEA}

ageratum, scented, yellow ............ per ounce $\$ 1.25$

millifolium kelwayi, magenta red ............. 3.00

ptarmica (Sneezewort) The Pearl, dible. white. " $\quad 3.40$

ACONITUM (Monk's Hood)

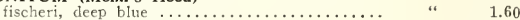

japonicum, bright blue ................ "4 1.80

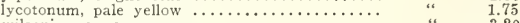

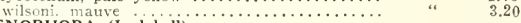

ADENOPHORA (Ladybeli)

lilifolia, fragrant pale blue $\ldots \ldots \ldots \ldots \ldots \ldots \ldots$ " $4 \ldots \ldots$

ADONIS

nolymorpha, purple blue $\ldots \ldots \ldots \ldots \ldots \ldots \ldots \ldots \ldots$ * $\quad 4.00$

aestivalis. red $\ldots \ldots \ldots \ldots$ per $1 / 2$ lb. $\$ 0.50$

vernalis, rich yellow $\ldots \ldots \ldots \ldots \ldots \ldots \ldots \ldots \ldots$ AGAPANTHUS

umbellatus, blue ........ per 1i oz. $\$ 0.90$

AGERATUM

mexicanum, Blue Perfection, large-fl........

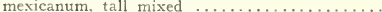

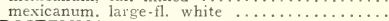

AGROSTEMMA

coeli-rosa (Rose of Heaven)..............

coronaria (Lychnis), Rose Champion ..........

ALTHEA

ficifolia, Antwerp Hollyhock, mixed ........

officinalis, Marsh Mallow, pink $\ldots . . .$.

ALYSSUM

argenteum, yellow

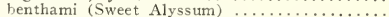

benthami compactum, Little Gem, white......

rostratum, yellow $\ldots \ldots \ldots \ldots \ldots \ldots \ldots \ldots \ldots$

saxatile compactum, golden ............

saxatile sulphureum,

ANCHUSA

sulphur yellow...$\ldots \ldots$ per $1 / 2$ oz. $\$ 2.50$

capensis. dark blue

2.50

ANDROSACE

cornopifolia, white $\ldots \ldots \ldots \ldots \ldots \ldots \ldots \ldots$

ANEMONE

coronaria, Chrysanthemum-dble. single mixed.

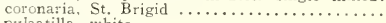

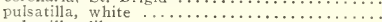

nulsatilla, lilac

ANTHEMIS

tintoria, yellow

ANTHERICUM (Liliastrum)

St. Brumn's Lily. pure white.. per $1 / 2$ oz. $\$ 1.20$

ANTIRRHINUM (Snapdragon)

nana, semi-dwarf mixed

nana. semi-dwarf mixed

AQUILEGIA

alpina, blue

canadensis, old rose with y $\ldots \ldots \ldots \ldots \ldots \ldots \ldots$

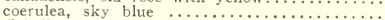

flabellata nana alba,

early white fl. ........ per $1 / 8$ oz. $\$ 1.80$

jaetschaui, large yellow peach red spurs.....

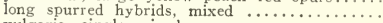

vulgaris, single mixed $\ldots \ldots \ldots \ldots \ldots \ldots \ldots \ldots \ldots \ldots$

vulgaris, double-fl. mixed

ARABIS (Rock Cress)

albida, white

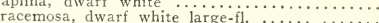

rosea, live rose-colored ..... per if $_{4}$ oz. $\$ 2 . \dot{2}$

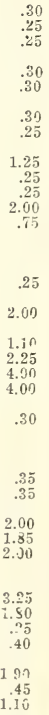


FLOWERING PLANT SEIDD-COnt

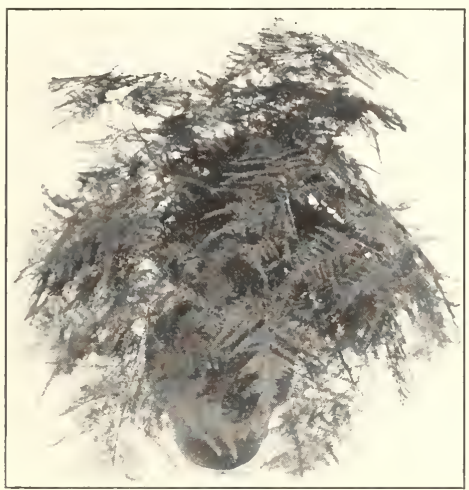

ASPARAGUS

plumosus nanus

per 10000 seed $\$ s n$

per 10000 seed 7.00

\section{ARCTOTIS}

ARENARIA Nim Lilte Dairy

ARNICA

AScintana, fragrant yell w .... per ${ }^{1}{ }_{2}$ oz. \$n. 70

ASCLEPIAS

ASPERULA

ASTER

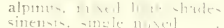

ASTILBE

arendsii gloria, dark is -

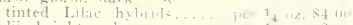

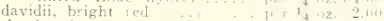

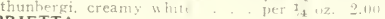

\section{AUBRIETTA}

deltridea bougainville?

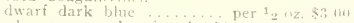

deltuidea graeca, dwatt likht blue.

deltoidea Leichtlinii. deep rose per is az \$2 at

deltriclea Whitewell Gem.

BAPTISIA

ey purple tinged blue.... per ${ }^{1 / 2}$ oz. 4. (n)

australis, deep lilue

\section{BELLIS}

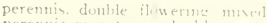

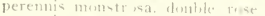

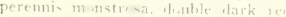

BLETILLA

hyacinthma

BOCCONIA

BROWALLIA

ratit luc

UPHTHALMUM

\section{CALANDRINIA}

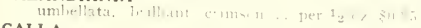

\section{CALLA}

CALLIOPSIS (Coreopsis)

per ${ }^{1}+16 . \$ 2.75$ 
FLOWERING PLANT SEED-COnt'd

\section{CAMPANULA}

grandiflora-see Platyccdon

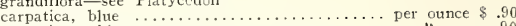

carpatica, white

medium (Canterbury Bells) single mixed ....

medium (Canterbury Bells), single blue .....

medium (Canterbury Bells), single lilac ......

medium (Canterbury Bells), single rose .....

medium (Canterbury Bells), single white .....

medium (Canterbury Bells) double blue ......

medium (Canterbury Bells) double white ....

wedium calycanthena, albo, white..........

medium calycanthema, blue $\ldots \ldots \ldots \ldots \ldots \ldots$

medium calycanthema rosea, rose $\cdots \cdots \cdots$ persicifolia grandiflora, white... per $1 / 2$ oz. $\$ 1.00$

speculum, mixed $\ldots \ldots \ldots \ldots \ldots \ldots \ldots \ldots \ldots \ldots \ldots \ldots \ldots \ldots \ldots \ldots$

indica new $1 \mathrm{~g}$. $\mathrm{fl}$, varieties mixed per $1 / 4 \mathrm{lb}, 2.75$

CARNATIONS (see Dianthus caryphyllos

\section{CATANANCHE}

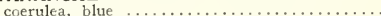

coerulea, white

\section{CELOSIA}

childsi, (Chinese Woolflower) mixed........

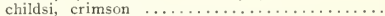

cristata (Cockscomb), dwarf nixed ...........

cristata, tall mixed $\ldots \ldots \ldots \ldots \ldots \ldots \ldots \ldots \ldots$

pyramidalis plumosa, mixed $\ldots \ldots \ldots \ldots \ldots \ldots$

\section{CENTAUREA}

cyanus, double blue, Bachelor Button.........

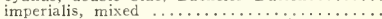

In colors, lilac, purple, rose, white........

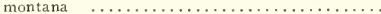

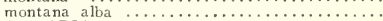

\section{CEPHALARIA}

tatarica. Yellow Giant Scabious............

CERASTINUM

tomentosum, creeping gray foliage ........

\section{CHELONE}

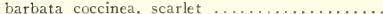

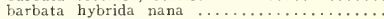

\section{CHRYSANTHEMUM}

coronarium, double flowering mixed.........

japonicum (A new Introduction) per $1 / 2$ oz. $\$ 2.00$

\section{CLARKIA}

elegans 11.-pl. alba, double-white ..........

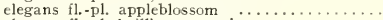

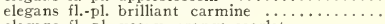

\section{CLEMATIS}

davidiana (Heracleaefolia),

fragrant, deep blue...... per $1 / 2$ oz. $\$ 2.50$

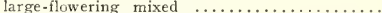

paniculata, fragrant white

CLEOME (Japanese Virgin Bower) .... \$3.00 per $1 \mathrm{~b}$.

\section{pungens}

pun

lachrymae (Job's Tear) ....... per 1b. $\$ 0.40$

\section{COLEUS}

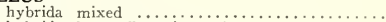

hybrida large flowering mixed $\ldots \ldots \ldots \ldots \ldots \ldots$

\section{COSMOS}

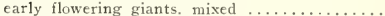

late flowering, double mixed $\ldots \ldots \ldots \ldots \ldots \ldots$.

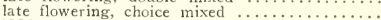

CRUCIANELLA

stylosa, bright purple

CYNOGLOSSUM

amabile, blue Chinese Forget-me-not........

DAHLIA (Trade packets $\$ 1.00$ )

double Cactus flowering. mixed $\ldots \ldots \ldots \ldots \ldots$ double Liliput small flowering, mixed .........

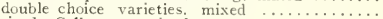

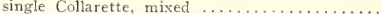

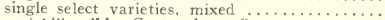
variabilis, dble. Cactus large-fl.

hybrids .............. per $1 / 2$ oz. 2.50 variabilis, Double Lilliput, small-fl. mixed.....

\section{DELPHINIUM}

belladonna, improved $\ldots \ldots \ldots \ldots \ldots \ldots \ldots$

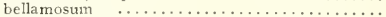

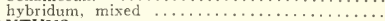

\section{DIANTHUS}

caryophyllus, (Marguerite Double Carnations),

chinensis (Pinks), double mixed $\ldots \ldots \ldots \ldots \ldots \ldots$

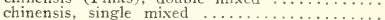

plumarius (Grass Pinks) single mixed....... 
FLOWERING PLANT SEED-Cont'd

DICENTRA (Trade packets $\$ 1.00$ )

exmma

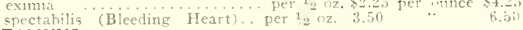

\section{DICTAMNUS}

iraxinella, pink .................... $\quad 1.00$

traxinella, white ...................... $1.0 \mathrm{C}$

DIGITALIS

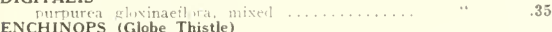

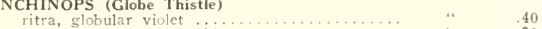

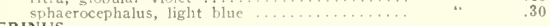

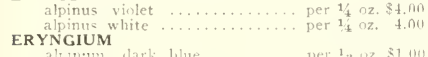

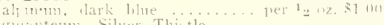

ERYSIMUM

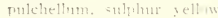

ESCHSCHOLTZIA

EULALIA (Misconthus)

jatonica

ageratotdes, white .......... per 30,7 रो र

$$
\text { ageratordes, }
$$

\section{EUPHORBIA}

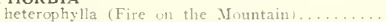

heterophy (Snche on the Mrountain .

FORGET-ME-NOT-see Myosotis

\section{GAILLARDIA}

grandifora, largen flowered mixed

grancliflora maxima. golden yellow

grandiflora sanguinea, purple red

grandiflora oculata, light colors with red center.

picta forenziana, double mixed

GENTIANA

acatilis, gentian blue ........ prer 1 , nz, \$1,25

lutea, vellow

purpurea, rosy-purplo

\section{GERBERA}

GEUM

cernium. scarlet

coccinium atrosanguineum, semi-dble. orange red coccinium. Mrs. Bradshaw, dble., brilliant red.

\section{GLOBULAR IA}

\section{GODETIA}

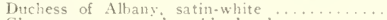

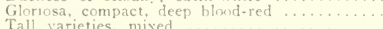

\section{GYPSOPHILA}

elegans, grandiflora alba, large-il.

paniculata

paniculata, double flowering

repens, dwarf creeper, fls. white

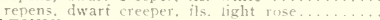

HELIANTHEMUM

mutabile

HELICHRYSUM

monstonsum, hint is mixed

HELIOPSIS

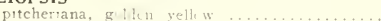

scabra zinniaeflora, semi-dble, yellow ...........

HELIOTROPIUM

lars. thwering, di ulile mixed ................ 1.09

\section{HELLEBORUS}

hybtulus, finest mixer]

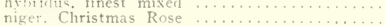

\section{HESPERIS}

n.tis nalis (Sweet Rocket), purple

\section{HEUCHERIA}

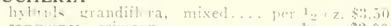

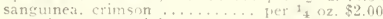
sanguinea, rose pink ........ per 1/4 oz. 2.00 sanguinea splendens, vermillion red.......... per ${ }^{1}{ }_{1}$ nz, 3.00

\section{HIBISCUS}

mutrhilis 
FLOWERING PLANT SEED-Cont'd

\section{INULA}

IRIS

royleana, large yellow $\ldots \ldots \ldots$ per $1 / 2 \mathrm{oz} . \$ 0.95$

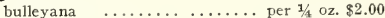

chrysographes $\ldots \ldots \ldots \ldots \ldots$ per $1 / 4$ oz. 1.75

clarkei $\ldots \ldots \ldots \ldots \ldots \ldots \ldots \ldots$ per $1 / 4$ oz. 2.50

ensata $\ldots \ldots \ldots \ldots \ldots \ldots$ per $1 / 4$ oz. 1.75

forresti $\ldots \ldots \ldots \ldots \ldots$ per $1 / 4$ oz. $\$ 2.50$

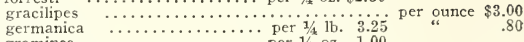

graminea $\ldots \ldots \ldots \ldots \ldots \ldots \ldots$ per $1 / 4$ oz. 1.00

japonica $\quad \cdots \cdots \cdots \cdots \cdots \cdots$ per $1 / 4$ oz. 1.75

kaempieri, collected from

cultivated plants ....... per $1 / 1$ lb. 3.00 koreana .............................. $1 / 45$

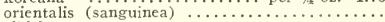

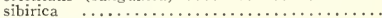

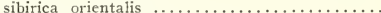

xiphiodes (English Iris),

dark blue (Royal Blue) $\ldots \ldots \ldots \ldots \ldots \ldots$

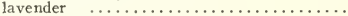

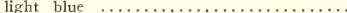

KOCHIA

tricophylla (Mexican Burning Bush)......... " .

KUDZU VINE

pueraria thunbergiana ........ per lb. $\$ 1.75$

\section{LARKSPUR}

stock flowered tall double mixed .......... "

\section{LATHYRUS}

latifolius, mixed

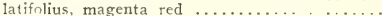

latifolius, pink

LAVANDULA

vera. Clean Seed

\section{LEONTIPODIUM}

alpinum, Edelweiss ........ per $1 / 8$ oz. $\$ 2.00$

\section{LIATRIS}

pycnostachya

scariosa

等,

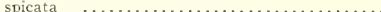

\section{LILIUM}

a mabile

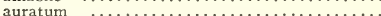

auratum platyphyllum (Macranthum) .......

backhouse hybrids $\ldots \ldots \ldots \ldots$ per $1 / 4$ nz. $\$ 5.00$

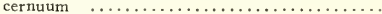

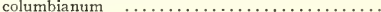

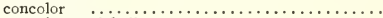

concolor pulchellum $\ldots \ldots \ldots \ldots \ldots \ldots \ldots \ldots \ldots$

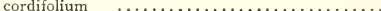

croceum $\quad \ldots \ldots \ldots \ldots \ldots \ldots \ldots \ldots \ldots \ldots \ldots \ldots \ldots$

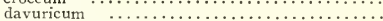

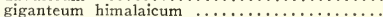

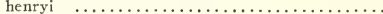

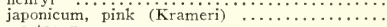

martagon

per $1 / 4$ oz. $\$ 5.00$

martagon dalmaticum ........ per $1 / 4$ oz

nepalense (Himalayan origin).. per $1 / 4$ oz. $\$ 2.00$ occidentalis

$\ldots \ldots \ldots \ldots \ldots \ldots \ldots \ldots \ldots \ldots$ per $1 / 4$ oz. 3.00

parryi $\ldots \ldots \ldots \ldots \ldots \ldots \ldots$ per $1 / 4$ oz. 3.00
philippinense formosanum $\ldots \ldots \ldots \ldots \ldots \ldots$

\section{LILIUM PHILIPPINENSE FORMOSANUM}

We also have available a small quantity of a strain specially bred by $T$. Sakata \& Co. It flowers a bit more quickly and of its blooms at least $60 \%$ are of the purest pure white.

Sakata's L. philippinense formosanum

$$
\$ 2.00 \text { per } 1 / 2 \text { oz. } \$ 4.00 \text { per oz. }
$$

regale

roezli

rubellum

$\ldots \ldots \ldots \ldots \ldots \ldots \ldots \ldots \ldots \ldots \ldots$

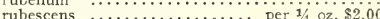

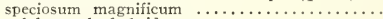

sulphur-gale hybrids,

(Sulphureum X Regale).... per 1/4 oz. 3.50

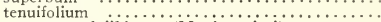

ventusum fujihirato (Maximowiczi)

wallichianum ........... per $1 / 4$ oz. $\$ 2.00$

washingtonianum $\ldots \ldots \ldots \ldots \ldots \ldots \ldots \ldots \ldots$ 
FLOWERING PLANT SEED-CON'd.

\section{LINARIA}

alpina, violet blotched orange...per is oz. $\$ 3.00$

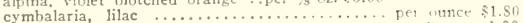

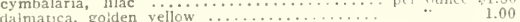

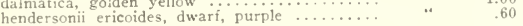

\section{LINUM}

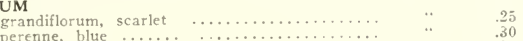

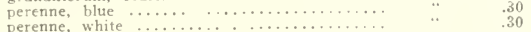

\section{LOBELIA}

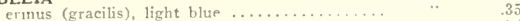

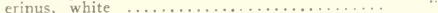

\section{LUNARIA}

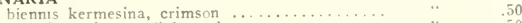

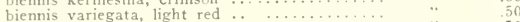

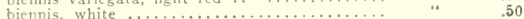

\section{LUPINUS}

annual varieties mixed ......... per $1 \mathrm{~b} . \$ 1.00$

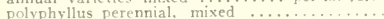

polyphyllus perennial, blue $\ldots \ldots \ldots \ldots \ldots \ldots$

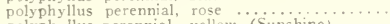

\section{LYCHNIS}

arkwrighti

chalcedonica, scarlet

chalcedonica, white

\section{LYTHRUM}

\section{MARIGOLD}

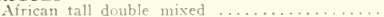
African tall aurantiaca, orange $\ldots \ldots \ldots \ldots \ldots \ldots$

\section{MATRICARIA}

eximia finlden Ball. twarf dble. fl..........

MESEMBRIANTHEMUM

crystallinum, Ice Plant

MIGNONETTE

odorata, large flowering, mixed $\ldots \ldots \ldots \ldots$.

\section{MIMOSA}

pudica. Sensitive Plant

tigrinns, vellow spotted

\section{MOMORDICA}

charantia (Balsam Pear)

MYOSOTIS

alpestris nana, dwari blue

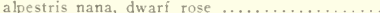

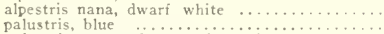

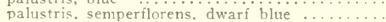

NICOTIANA (Flowering Tobacco)

hybrida. choice mixed

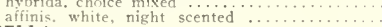

\section{NIGELLA}

ramascena, double mixed

\section{OENTHERA}

lamarkıana, large Primrose-flower

5

PAEONIA

chinensis, double-fl. mixed ..... per lb. $\$ 3.75$ PANSY - see also Viola

Bedding varieties, mixed ...... per lb. $\$ 4,00$

\section{PENSTEMON}

digitalis. white $\ldots \ldots \ldots \ldots \ldots \ldots \ldots \ldots \ldots \ldots \ldots \ldots \ldots \ldots$
glaber, bright blue $\ldots \ldots \ldots \ldots \ldots \ldots \ldots \ldots \ldots \ldots \ldots \ldots$
glaber roseus, pinkish rose $\ldots \ldots \ldots \ldots \ldots \ldots \ldots \ldots \ldots$
oratus, blue $\ldots \ldots \ldots \ldots \ldots \ldots \ldots \ldots \ldots$

\section{PETUNIA}

hybrida, mixed

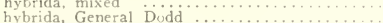

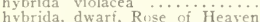

hybrida, dwarf, Rosy Uorn

hybrida, dwart. (nana) mixed

hybrida grandiflora, large-fl. mixed

hybrida gril fringed mxd... per $1 . .17$ \&3. il

hybrida, Balcony, large-fl. mixed

\section{PHLOX}

decussata, mixed

drummoncli cuspidata (Star Phlox) mixed

drummondi, grandiflora finest mixed

\section{PLATYCODON}


FLOWERING PLANT SEED-COnt'd.

\section{POPPY}

nudicaule. (Iceland Poppy), mixed .......... per ounce $\$ .50$ nudicaule, double mixed.$\ldots \ldots \ldots \ldots \ldots \ldots \ldots$ orientale, mixed $\ldots \ldots \ldots \ldots \ldots \ldots \ldots \ldots \ldots$ orientale, glowing red $\ldots \ldots \ldots \ldots \ldots \ldots \ldots$. orientale, orange scarlet $\ldots \ldots \ldots \ldots \ldots \ldots \ldots$ orientale, scarlet

\section{PORTULACA}

single flowering mixed

PRIMULA

a uricula, finest mixed $\ldots \ldots \ldots \ldots \ldots \ldots \ldots$

japonica, mixed m.

veris (Cowslip), mixed ...... per $1 / 2$ oz. 1.75

\section{PRUNELLA}

\section{grandifiora, blue}

\section{PYRETHRUM}

roseum hybridum, single mixed

\section{RANUNCULUS}

asiaticus superbissimus, mixed

RUDBECKIA

purpurea (Echinacea), purple-red

SALPIGLOSSIS

emperor, exquisite mixed

SALVIA

bonfire, early blooming

SAPONARIA

ocymoides, rose $\ldots \ldots \ldots \ldots \ldots \ldots \ldots \ldots \ldots$

\section{SAXIFRAGA}

azoon, white silver-braided.... per $1 / 3$ oz. $\$ 2.25$ cordifolia, rose $\ldots \ldots \ldots \ldots \ldots$ per $1 / 8$ oz. 3.00 hypnoides, white mosslike..... per $1 / 8$ oz. 2.50 SCABIOSA

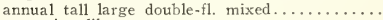

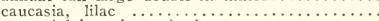

SEDUM

acre, Golden Moss ......... per $1 / 8$ oz. $\$ 1.60$

SIDALCEA

Rosy Gem

SILENE

alpestris, intense white ..... per $1 / 2$ oz. 1.50 schafta, magenta .......... per $1 / 2$ oz. 1.25

SPIREA arungus, Clean Seed $\ldots \ldots \ldots \ldots \ldots \ldots \ldots \ldots \ldots \ldots \ldots \ldots \ldots \ldots$

STATICE

incana, small white flowers.. per $1 / 2$ oz. .50 latifolia $\ldots \ldots \ldots \ldots \ldots \ldots \ldots$ per $1 / 2$ oz. 1.25 sareptana, dark blue ......... per $1 / 2$ oz. 1.50

STOCKS

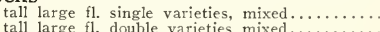

STOKESIA (Cornflower Aster)

cyanea, blue

cyanea, white

SWEET WILLIAM

double flowering mixed

single flowering, mixed $\ldots \ldots \ldots \ldots \ldots \ldots \ldots \ldots$

THERMOPSIS

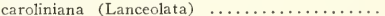

TORENIA

fournieri, blue

single flowering white

TRITOMA (Kniphofia)

hybrida in elegant variety.... per $1 / 4$ oz. $\$ 1.25$

uvaria grandiflora

VERBENA

hybrida mixed

VERONICA

longifolia

pulchella, dwarf navy blue.... per $1 / 2$ oz. $\$ 2.00$
repens, early dwarf, white and

light blue .............. per $1 / 4 \mathrm{oz}, 2.00$

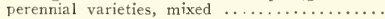

VINCA

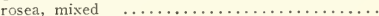

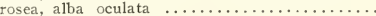

rosea, alba pura, pure white...$\ldots \ldots \ldots \ldots$.

VIOLA-see also Pansy

Blue Perfection

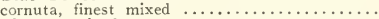

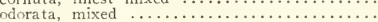

\section{YUCCA}

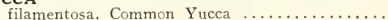

\section{ZINNIA}

elegans, tall double mixed $\ldots \ldots \ldots \ldots \ldots \ldots$

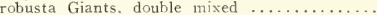

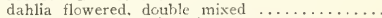

liliput dwarf, double mixed

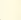

